\title{
Anxiety, distress and anger among British nationals in Japan following the Fukushima nuclear accident
}

\author{
G. James Rubin, Richard Amlôt, Simon Wessely and Neil Greenberg
}

\section{Background}

The 2011 earthquake and tsunami in Japan caused a meltdown at the Fukushima nuclear power plant.

\section{Aims \\ To quantify emotional responses among British nationals in Japan and to assess whether perceptions about the incident or accessing information about it were associated with responses.}

\section{Method}

A total of 284 participants randomly selected from official records completed a survey that included instruments to measure emotional responses.

\section{Results}

In total, $16 \%$ met the criteria for distress, $29.7 \%$ reported high anxiety relating to the incident and $30.4 \%$ reported high anger. Perceptions that strongly predicted these outcomes included feeling uncertain, being unable to rule out harmful exposure, and believing that exposure would have severe or hidden health effects or be difficult to detect. Using information sources was associated with higher emotional outcome, particularly for sources perceived to have low credibility.

\section{Conclusions}

Reducing uncertainty and improving the credibility of information is essential in reducing the psychological impact of radiological disasters.

\section{Declaration of interest}

None.
In 2011, Japan experienced a catastrophic earthquake and tsunami that triggered meltdowns in some of the reactors at the Fukushima nuclear power plant. Efforts to contain this crisis continued for months. A $20 \mathrm{~km}$ exclusion zone around the plant was set up by the Japanese government, while other countries, including Britain, advised their citizens to stay at least $60 \mathrm{~km}$ away and issued them with iodine tablets to take should the situation worsen. The British Foreign and Commonwealth Office (FCO) attempted to contact British nationals in Japan, to help them relocate to safer areas if necessary and to communicate the risks associated with the evolving situation at Fukushima. Teleconferences were arranged for British nationals to discuss the situation directly with the British government's chief scientific advisor. Although formal advice to leave the country was not given, British nationals were advised to 'consider leaving. In this paper, we quantify levels of distress, anxiety and anger among a random sample of British nationals who were in contact with the FCO following the disaster. We assess whether their emotional reactions were associated with their uncertainty about the incident, their perceptions about radiation or their use of specific information sources, and whether these associations were dependent on how systematically a participant thought about the incident ${ }^{1}$ or how credible they found an information source. ${ }^{2}$ We also describe those aspects of the FCO's response that were perceived by British nationals as being helpful or unhelpful.

\section{Method}

\section{Design and participants}

We used a cross-sectional survey to measure all variables. The wording and result for each survey item is given in the online supplement to this paper. We randomly selected participants from a database maintained by the FCO of all contacts made with members of the public about the disaster. We included people who were at least 18 years old and who held British nationality only. We excluded those enquiring about the safety of someone else.

\section{Outcome variables}

We measured distress using the 12-item General Health Questionnaire (GHQ-12) $)^{3}$ and classed participants as experiencing distress if they scored 4 or more. We measured anger using the 'feeling angry' subscale of the State-Trait Anger Expression Inventory- $2^{4}$ and anxiety using the 6-item State-Trait Anxiety Inventory. ${ }^{5}$ We asked participants to rate emotions over the past week, when thinking about the radiation leak. We defined high anger as a score of 11 or more $^{4}$ and high anxiety as a score of 18 or more. $^{6}$

\section{Demographic variables and exposure to the disaster}

We recorded each participant's age, gender, ethnicity, highest educational qualification, parental status and the presence of any long-standing illness, disability or infirmity.

We asked participants what region of Japan they were in when the earthquake struck, whether they had left Japan afterwards, and if so, why. We also asked whether they had experienced any of 16 potentially upsetting events following the disaster. ${ }^{7}$

\section{Perceptions about the radiation incident}

We asked participants 'How much radiation you believe you were exposed to as a result of the accident at the Fukushima nuclear power plant?' Possible answers were 'none' or 'some.' Those who responded 'some' where asked to quantify this as 'definitely not enough to affect someone's health', 'probably not enough to affect someone's health', 'may or may not be enough to affect someone's health', 'probably enough to affect someone's health' or 'definitely enough to affect someone's health'. 
We used the Perceptions AbouT Hazardous Substances (PATHS) questionnaire to assess perceptions about the radioactive material released during the incident. ${ }^{8}$ The PATHS includes six scales which assess the perceived mysteriousness of a substance, the severity of its effects, whether it produces hidden health effects, whether the substance is easy to detect, whether it is possible to discriminate the symptoms of exposure from those of other illnesses and whether certain groups are particularly at-risk. The PATHS also includes five items measuring the perceived mechanisms through which a substance might affect health and one item assessing the perceived delay between exposure and the first symptoms occurring. In this study, items were worded so as to relate to 'the kind of radiation that was released from the Fukushima nuclear power plant.8 Questions about severity asked participants to consider the radiation levels likely to be present just outside the $60 \mathrm{~km}$ exclusion zone.

Participants described whether they had felt uncertain over the past week when thinking about the radiation incident using the same response options as for the anger and anxiety inventories. ${ }^{4,5}$

We asked participants to describe how they mentally processed information about the incident using an adapted version of the systematic Information Processing Questionnaire. ${ }^{1}$ Individuals scoring highly on systematic processing are characterised as making a strong effort to understand and think about information.

\section{Information sources about the incident}

Participants rated 17 types of information source concerning the radiation incident as 'did not use', 'used, received no information', 'used, received some information' or 'used, received a lot of information', and to rate the credibility of information about the incident from the British government, the Japanese government, the British media and the Japanese media using Meyer's credibility index. ${ }^{2,9}$

\section{Perceptions about the British Embassy and FCO}

We asked participants to rate the 'help you received from the Embassy or Foreign Office, after the disaster' and to describe in their own words one thing that the Embassy or FCO could have done better and one thing that they did well.

\section{Procedure}

The FCO sent invitations on our behalf to 607 people. Where an email address was available, an initial information email was followed by a second email containing a link to our online questionnaire and a reminder 2 weeks later. Where only a postal address was available, a letter was sent instead. Participants were told that we would donate $\mathfrak{E} 5$ to charity if they completed the questionnaire. Data were collected during December 2011.

\section{Analyses}

For questions allowing a free-text response, we combined free-text answers into an existing closed response category if this seemed appropriate or used thematic analysis to code them into new categories.

An exploratory factor analysis of the PATHS items intended for use as scales revealed a factor structure that almost exactly replicated that found for other forms of hazardous substance, ${ }^{8}$ although only two items, rather than three, loaded onto the 'easy to spot exposure' scale and no scale could be formed for the items which asked participants to identify which groups of people were most at risk from exposure. Scores for PATHS scales were calculated as the mean of those items which loaded onto them.
Scores for individual items which used five-point Likert scales were treated as continuous variables. Responses for the single PATHS item relating to the perceived latency between exposure and symptom onset were recoded as ' 24 hours to 2 days', ' 2 days to 6 months' or 'more than 6 months'.

We dichotomised scores on the systematic processing and credibility measures using median splits.

We used binary logistic regressions to calculate odds ratios for the associations between predictor and outcome variables. For information sources that represented discrete events such as contacting someone from the Embassy or attending the teleconference with the chief scientific advisor, we compared people who had done this with those who had not. For information sources which people might consult on a more regular basis (e.g. use of mass media), we compared those who had received a little information in this way with those who received a lot of information. We included all demographic variables as potential confounders, except in those regressions testing the association between PATHS scores and outcome variables. For those, we considered that PATHS variables might mediate any link between demographics and psychological outcomes, obviating the need to adjust for demographics. To test for potential interactions between PATHS scores and systematic information processing, or between using an information source and the credibility of that source, we included interaction terms in the regressions. Where significant interactions were found we calculated the association between predictor and outcome variables separately for people who were high or low on systematic processing or credibility.

We analysed qualitative responses about what the FCO and British Embassy did well or could have done better by combining responses to these questions and using thematic analysis to group similar responses into the themes and subthemes.

\section{Results}

We received 'undeliverable' or 'out of office' notifications for 44 invitations to our survey. In total, 299 participants began the survey and 284 completed it. Response rates therefore vary from $53.1 \%$ to $50.4 \%$. Participants were predominantly male $(n=215$, $71.9 \%)$, White $(n=275,91.7 \%)$ and well-educated, with 257 people $(85.9 \%)$ having completed a degree. Although no formal data exist, it seems plausible that these demographics are representative for British visitors to Japan. Other demographics are shown in Table 1. Most participants $(n=205,68.5 \%)$ had been in Tokyo or Kanto province when the earthquake struck. One hundred and one participants $(33.8 \%)$ reported leaving Japan following the disaster, for the reasons summarised in Table 2. Of those who stayed, 31 (15.7\%) would have preferred to leave.

Overall, 46 participants $(16.1 \%)$ met the criteria for distress, $85(29.7 \%)$ for high anxiety and 87 (30.4\%) for high anger.

\section{Association between demographics or exposure to potentially upsetting events and distress, anxiety or anger}

Fewer than $2 \%$ of the sample $(n<6)$ had experienced most of the potentially upsetting experiences that we asked about. However, 91 participants $(30.6 \%)$ reported having been scared that they would be killed or seriously injured, 146 (49.2\%) were scared that a loved one or friend would be killed or seriously injured, 27 (9.1\%) reported losing personal property or belongings and 30 (10.1\%) reported that their home was damaged. Tables 1 and 3 show the associations between the demographic or exposure variables and our outcomes. No demographic variable showed 


\begin{tabular}{|c|c|c|c|c|c|}
\hline Variable & $\begin{array}{c}\text { Participants, } \\
n(\%)\end{array}$ & $\begin{array}{c}\text { Experiencing } \\
\text { distress, } n(\%)\end{array}$ & OR $(95 \% \mathrm{Cl})$ & $\begin{array}{l}\text { Experiencing } \\
\text { anxiety, } n(\%)\end{array}$ & OR $(95 \% \mathrm{Cl})$ \\
\hline Female & $84(28.1)$ & $17(21.5)$ & $1.7(0.9-3.3)$ & $37(46.8)$ & $2.9(1.7-5.0)$ \\
\hline Male & $215(71.9)$ & $29(14.0)$ & Reference & $48(23.2)$ & Reference \\
\hline \multicolumn{6}{|l|}{ Age, years } \\
\hline $18-30$ & $75(25.1)$ & $14(19.4)$ & $1.2(0.5-2.9)$ & $23(31.9)$ & $1.1(0.5-2.2)$ \\
\hline $31-36$ & $75(25.1)$ & $14(18.9)$ & $1.1(0.5-2.8)$ & $25(33.8)$ & $0.7(0.3-1.4)$ \\
\hline $37-45$ & 79 (26.4) & $7(9.6)$ & $0.5(0.2-1.5)$ & $18(24.7)$ & $0.8(0.4-1.7)$ \\
\hline$\geqslant 46$ & $70(23.4)$ & $11(16.4)$ & Reference & $19(28.4)$ & Reference \\
\hline \multicolumn{6}{|l|}{ Ethnicity } \\
\hline Other & $24(8.0)$ & $3(13.0)$ & $0.8(0.2-2.7)$ & $9(39.1)$ & $1.6(0.7-3.8)$ \\
\hline White & $275(92.0)$ & $43(16.3)$ & Reference & $76(28.9)$ & Reference \\
\hline \multicolumn{6}{|l|}{ Parental status } \\
\hline Has child aged $\leqslant 5$ years & $77(25.7)$ & $13(17.6)$ & $1.0(0.5-2.1)$ & $22(29.7)$ & $1.0(0.5-1.8)$ \\
\hline Has child aged $6-16$ years, but none aged $\leqslant 5$ & $36(12.0)$ & $2(6.3)$ & $0.3(0.1-1.4)$ & $9(28.1)$ & $0.9(0.4-2.1)$ \\
\hline Has no children, or children over 16 years only & $187(62.3)$ & $31(17.2)$ & Reference & $54(30.0)$ & Reference \\
\hline \multicolumn{6}{|l|}{ Education } \\
\hline GCSE or A-level & $42(14.0)$ & $8(19.5)$ & $1.3(0.6-3.1)$ & $11(26.8)$ & $0.8(0.4-1.8)$ \\
\hline Degree level or higher & $257(86.0)$ & $38(15.5)$ & Reference & $74(30.2)$ & Reference \\
\hline \multicolumn{6}{|l|}{ Long-standing illness } \\
\hline Present & $24(8.0)$ & $6(25.0)$ & $1.9(0.7-4.9)$ & $8(33.3)$ & $1.2(0.5-2.9)$ \\
\hline Absent & $275(92.0)$ & $40(15.3)$ & Reference & $77(29.4)$ & Reference \\
\hline \multicolumn{6}{|l|}{ Home was damaged } \\
\hline Yes & $30(10.1)$ & $10(35.7)$ & $3.5(1.4-8.6)^{\mathrm{a}}$ & $15(53.6)$ & $2.7(1.1-6.1)^{\mathrm{a}}$ \\
\hline No & $267(89.9)$ & $36(14.0)$ & Reference & $70(27.1)$ & Reference \\
\hline \multicolumn{6}{|l|}{ Lost property or belongings } \\
\hline Yes & $27(9.1)$ & $6(24.0)$ & $1.8(0.6-4.9)^{\mathrm{a}}$ & $7(28.0)$ & $0.9(0.3-2.2)^{a}$ \\
\hline No & $270(90.9)$ & $40(15.3)$ & Reference & $78(29.9)$ & Reference \\
\hline \multicolumn{6}{|l|}{ Scared that loved one would be killed or hurt } \\
\hline Yes & $146(48.7)$ & $31(22.3)$ & $2.6(1.3-5.2)^{\mathrm{a}}$ & $51(36.7)$ & $2.2(1.3-3.9)^{\mathrm{a}}$ \\
\hline No & $151(50.3)$ & $15(10.2)$ & Reference & $34(23.1)$ & Reference \\
\hline \multicolumn{6}{|l|}{ Scared that self would be killed or hurt } \\
\hline Yes & $91(30.6)$ & $20(23.5)$ & $2.1(1.1-4.1)^{\mathrm{a}}$ & $41(48.2)$ & $3.1(1.8-5.5)^{a}$ \\
\hline No & $206(69.4)$ & $26(12.9)$ & Reference & $44(21.9)$ & Reference \\
\hline
\end{tabular}

any association with distress or anger, although women were more likely to experience anxiety than men. Having suffered damage to one's home was associated with greater likelihood of distress and anxiety, whereas having been scared that oneself or one's loved ones would be hurt or killed was associated with distress, anxiety and anger.

\section{Perceptions about the radiation incident}

One hundred and forty-seven participants believed that they had been exposed to no radiation or definitely not enough to affect health. The remaining $150(50.5 \%)$ believed that they had been exposed to some radiation, but could not definitely rule out health effects. These participants were more likely to report distress (odds ratio $(\mathrm{OR})=2.6,95 \% \mathrm{CI} 1.3-5.1)$, anxiety $(\mathrm{OR}=2.7,95 \%$ CI 1.6-4.5) and anger $(\mathrm{OR}=2.8,95 \%$ CI 1.7-4.8). Adjusting for demographic variables did not affect these associations.

When asked whether they felt uncertain when thinking about the radiation incident, 66 participants $(23.1 \%)$ responded 'very much', 74 (25.9\%) responded 'somewhat', 87 (30.4\%) replied 'moderately' and 59 (20.6\%) replied 'not at all'. Participants responding 'very much' were substantially more likely than those responding 'not at all' to experience distress $(\mathrm{OR}=7.4,95 \% \mathrm{CI}$ 2.4-22.9), anxiety $(\mathrm{OR}=23.1,95 \%$ CI 8.1-66.3) and anger ( $\mathrm{OR}=4.8,95 \%$ CI 2.2-10.4). A large association was also noted for being 'somewhat' uncertain compared with 'not at all' for anxiety ( $\mathrm{OR}=5.2,95 \%$ CI 1.8-14.6). No other comparisons were significant. Adjusting for demographic variables did not affect these associations.
Mean scores for the PATHS scales and items, and their associations with the outcome variables, are shown in Table 4. Distress was associated with stronger beliefs that exposure might cause hidden health effects (for people scoring low for systematic processing) and that unwashed food posed a risk. Anxiety was associated with a stronger belief that: the radiation was mysterious; the effects of exposure would be severe; the effects

\begin{tabular}{|c|c|}
\hline Reason & $\begin{array}{l}\text { Participants } \\
\text { giving this } \\
\text { reason, } n(\%)\end{array}$ \\
\hline I was concerned about the radiation leak & $62(61.4)$ \\
\hline My friends or relatives wanted me to leave & $40(39.6)$ \\
\hline $\begin{array}{l}\text { My visit to Japan was over anyway, for reasons } \\
\text { not connected to the earthquake }\end{array}$ & $26(25.7)$ \\
\hline I was concerned there might be more earthquakes & $24(23.8)$ \\
\hline My holiday or business plans had been affected & $11(10.9)$ \\
\hline $\begin{array}{l}\text { Recommendation or requirement by employer } \\
\text { (e.g. 'My partner's company advised us to leave } \\
\text { and offered to pay our expenses') }\end{array}$ & $6(5.9)$ \\
\hline $\begin{array}{l}\text { I thought the British Embassy were advising people } \\
\text { to leave }\end{array}$ & $8(7.9)$ \\
\hline \multicolumn{2}{|c|}{$\begin{array}{l}\text { a. Participants were able to give more than one response. } \\
\text { b. This option was not offered to participants. Responses are derived from free-text } \\
\text { answers to the 'other reason' option. }\end{array}$} \\
\hline
\end{tabular}


of exposure might be hidden; young children or pregnant women were more at risk; and breathing contaminated air, eating washed or unwashed food and touching radioactive substances posed a risk. Anger was associated with believing that: the effects of exposure would be severe (high systematic processing group only); the effects of exposure might remain hidden; young children or pregnant women were more at risk; and that health could be affected by breathing contaminated air or eating washed or unwashed food. Anger was also associated with less belief that exposure would be easy to detect (low systematic processing group only). No significant associations were found between any outcome variable and the perceived latency between exposure and first symptoms (all $P>0.10$ ).

\section{Information sources about the incident}

Table 5 shows the frequency with which different information sources were used. The British government was considered the most credible source of information about the leak (mean credibility score 3.5 (s.d. $=0.8)$; scale range 1 'no credibility' to 5 'high credibility'), followed by the Japanese media (mean $=2.6$, s.d. $=0.8$ ), the British media $($ mean $=2.4$, s.d. $=0.9$ ) and the Japanese government $($ mean $=2.2$, s.d. $=0.8)$.

Table 6 shows the associations between using information sources and the outcome variables, adjusting for demographic variables. Low sample size prevented us from analysing the effects of speaking to a general practitioner. Distress was associated with listening to the teleconference with the chief scientific advisor (among participants who felt the British government had low credibility) and use of any British government website. Anxiety was associated with reading any official Japanese government website. Anger was associated with using any British media source (among participants who felt the British media had low credibility), reading any official Japanese government website and using any form of interpersonal communication.

\section{Perceptions about the FCO}

Overall, 23 participants said that they could not rate how helpful the British Embassy or FCO had been, 74 (28.2\%) rated their help as excellent, $117(44.7 \%)$ as good, $50(19.1 \%)$ as average and 21 $(8.0 \%)$ as poor.

The qualitative data identified seven important elements of the FCO's response (Table 7). Two related to the way British nationals had been contacted and the smooth running of the help offered with transport out of Japan. These are not considered in detail here. The remaining five themes covered information about radiation, provision of travel advice, provision of iodine tablets, provision of reassurance and intervening with the media. Of these, providing information about the radiation leak was the most frequently mentioned, with information being expected on issues such as 'the health risk (long and short term)', 'simplification of radiation measurements', sources of radiation 'in clouds ... in rain ... in food' and the appropriateness of actions that people could

\begin{tabular}{|c|c|c|c|}
\hline Variable & $\begin{array}{c}\text { Participants } \\
n(\%)\end{array}$ & $\begin{array}{c}\text { Participants } \\
\text { experiencing anger } \\
n(\%)\end{array}$ & OR $(95 \% \mathrm{Cl})$ \\
\hline Female & $84(28.1)$ & $27(34.2)$ & $1.3(0.7-2.2)$ \\
\hline Male & $215(71.9)$ & $60(29.0)$ & Reference \\
\hline \multicolumn{4}{|l|}{ Age, years } \\
\hline $18-30$ & $75(25.1)$ & $12(16.7)$ & $0.4(0.2-0.9)$ \\
\hline $31-36$ & $75(25.1)$ & $32(43.2)$ & $1.5(0.7-2.9)$ \\
\hline $37-45$ & $79(26.4)$ & $20(27.4)$ & $0.7(0.4-1.5)$ \\
\hline$\geqslant 46$ & $70(23.4)$ & $23(34.3)$ & Reference \\
\hline \multicolumn{4}{|l|}{ Ethnicity } \\
\hline Other & $24(8.0)$ & $7(30.4)$ & $1.0(0.4-2.5)$ \\
\hline White & $275(92.0)$ & $80(30.4)$ & Reference \\
\hline \multicolumn{4}{|l|}{ Parental status } \\
\hline Has child aged $\leqslant 5$ years & $77(25.7)$ & $25(33.8)$ & $1.3(0.7-2.3)$ \\
\hline Has child aged $6-16$ years, but none aged $\leqslant 5$ years & $36(12.0)$ & $11(34.4)$ & $1.3(0.6-2.9)$ \\
\hline Has no children, or children over 16 years only & $187(62.3)$ & $51(28.3)$ & Reference \\
\hline \multicolumn{4}{|l|}{ Education } \\
\hline GCSE or A-level & $42(14.0)$ & $15(36.6)$ & $1.4(0.7-2.8)$ \\
\hline Degree level or higher & $257(86.0)$ & $72(29.4)$ & Reference \\
\hline \multicolumn{4}{|l|}{ Long-standing illness } \\
\hline Present & $24(8.0)$ & $11(45.8)$ & $2.1(0.9-4.8)$ \\
\hline Absent & $275(92.0)$ & $76(29.0)$ & Reference \\
\hline \multicolumn{4}{|l|}{ Home was damaged } \\
\hline Yes & $30(10.1)$ & $10(35.7)$ & $1.0(0.4-2.3)^{a}$ \\
\hline No & $267(89.9)$ & $77(29.8)$ & Reference \\
\hline \multicolumn{4}{|l|}{ Lost property or belongings } \\
\hline Yes & $27(9.1)$ & $10(40.0)$ & $1.4(0.6-3.5)^{a}$ \\
\hline No & $270(90.9)$ & $77(29.5)$ & Reference \\
\hline \multicolumn{4}{|l|}{ Scared that loved one would be killed or hurt } \\
\hline Yes & $146(48.7)$ & $51(36.7)$ & $1.8(1.1-3.1)^{\mathrm{a}}$ \\
\hline No & $151(50.3)$ & $36(24.5)$ & Reference \\
\hline \multicolumn{4}{|l|}{ Scared that self would be killed or hurt } \\
\hline Yes & $91(30.6)$ & $34(40.0)$ & $1.8(1.01-3.1)^{\mathrm{a}}$ \\
\hline No & $206(69.4)$ & $53(26.4)$ & Reference \\
\hline
\end{tabular}




\begin{tabular}{|c|c|c|c|c|}
\hline Variable $^{a}$ & Mean (s.d.) $)^{b}$ & $\begin{array}{l}\text { Distress } \\
\text { OR }(95 \% \mathrm{Cl})\end{array}$ & $\begin{array}{l}\text { Anxiety } \\
\text { OR }(95 \% \mathrm{Cl})\end{array}$ & $\begin{array}{l}\text { Anger } \\
\text { OR }(95 \% \mathrm{Cl})\end{array}$ \\
\hline $\begin{array}{l}\text { Mysteriousness } \\
\text { High systematic processing } \\
\text { Low systematic processing }\end{array}$ & $\begin{array}{l}3.0(1.0) \\
3.0(1.1) \\
3.0(1.0)\end{array}$ & $\begin{array}{l}1.1(0.8-1.5) \\
\text { No interaction } \\
\text { No interaction }\end{array}$ & $\begin{array}{l}1.5(1.2-2.0) \\
\text { No interaction } \\
\text { No interaction }\end{array}$ & $\begin{array}{l}\text { See interaction } \\
1.3(0.9-1.8) \\
0.7(0.4-1.0)\end{array}$ \\
\hline $\begin{array}{l}\text { Severity } \\
\text { High systematic processing } \\
\text { Low systematic processing }\end{array}$ & $\begin{array}{l}2.8(0.8) \\
3.0(0.8) \\
2.7(0.8)\end{array}$ & $\begin{array}{l}1.2(0.8-1.7) \\
\text { No interaction } \\
\text { No interaction }\end{array}$ & $\begin{array}{l}2.8(1.9-4.0) \\
\text { No interaction } \\
\text { No interaction }\end{array}$ & $\begin{array}{l}\text { See interaction } \\
2.4(1.5-3.9) \\
1.0(0.6-1.6)\end{array}$ \\
\hline $\begin{array}{l}\text { Hidden health effects may exist } \\
\text { High systematic processing } \\
\text { Low systematic processing }\end{array}$ & $\begin{array}{l}4.0(0.7) \\
4.1(0.6) \\
3.8(0.7)\end{array}$ & $\begin{array}{l}\text { See interaction } \\
1.2(0.6-2.4) \\
3.6(1.6-8.2)\end{array}$ & $\begin{array}{l}2.2(1.5-3.4) \\
\text { No interaction } \\
\text { No interaction }\end{array}$ & $\begin{array}{l}1.9(1.2-2.8) \\
\text { No interaction } \\
\text { No interaction }\end{array}$ \\
\hline $\begin{array}{l}\text { Exposure is easy to detect } \\
\text { High systematic processing } \\
\text { Low systematic processing }\end{array}$ & $\begin{array}{l}1.4(0.6) \\
1.4(0.6) \\
1.4(0.6)\end{array}$ & $\begin{array}{l}0.9(0.5-1.5) \\
\text { No interaction } \\
\text { No interaction }\end{array}$ & $\begin{array}{l}0.7(0.4-1.1) \\
\text { No interaction } \\
\text { No interaction }\end{array}$ & $\begin{array}{c}\text { See interaction } \\
0.9(0.5-1.7) \\
0.3(0.1-0.8)\end{array}$ \\
\hline Possible to discriminate symptoms from other illnesses & $3.1(0.8)$ & $1.1(0.7-1.7)$ & $0.8(0.6-1.2)$ & $1.2(0.8-1.6)$ \\
\hline Under 5-year-olds more at risk & $4.3(0.8)$ & $1.3(0.9-2.1)$ & $1.6(1.1-2.3)$ & $1.7(1.2-2.4)$ \\
\hline Pregnant women more at risk & $4.1(0.8)$ & $1.2(0.8-1.9)$ & $2.2(1.5-3.2)$ & $1.7(1.2-2.4)$ \\
\hline Over 65-year-olds more at risk & $2.8(1.1)$ & $1.1(0.8-1.5)$ & $1.0(0.8-1.3)$ & $0.9(0.7-1.2)$ \\
\hline Those with pre-existing illness more at risk & $3.2(0.9)$ & $1.2(0.9-1.8)$ & $1.1(0.9-1.5)$ & $1.2(0.9-1.6)$ \\
\hline $\begin{array}{l}\text { You can be affected by breathing in air containing } \\
\text { radioactive substances }\end{array}$ & $4.2(0.8)$ & $1.5(0.9-2.3)$ & $1.5(1.03-2.1)$ & $1.9(1.3-2.8)$ \\
\hline $\begin{array}{l}\text { You can be affected by eating food contaminated with } \\
\text { radioactive substances that has not been washed }\end{array}$ & $4.4(0.7)$ & $1.8(1.02-3.1)$ & $2.1(1.3-3.2)$ & $2.3(1.4-3.6)$ \\
\hline $\begin{array}{l}\text { You can be affected by eating food contaminated with } \\
\text { radioactive substances that has been washed } \\
\text { High systematic processing } \\
\text { Low systematic processing }\end{array}$ & $\begin{array}{l}4.0(0.9) \\
4.1(0.9) \\
3.88(0.9)\end{array}$ & $\begin{array}{l}\text { See interaction } \\
1.4(0.8-2.4) \\
1.3(0.7-2.2)\end{array}$ & $\begin{array}{l}1.5(1.1-2.0) \\
\text { No interaction } \\
\text { No interaction }\end{array}$ & $\begin{array}{l}1.4(1.1-1.9) \\
\text { No interaction } \\
\text { No interaction }\end{array}$ \\
\hline You can be affected if you touch radioactive substances & $3.7(1.0)$ & $1.3(0.9-1.8)$ & $1.3(1.04-1.7)$ & $1.1(0.8-1.3)$ \\
\hline $\begin{array}{l}\text { You can be affected if you come into close contact with } \\
\text { someone who was in the exclusion zone }\end{array}$ & $2.5(1.2)$ & $1.2(0.9-1.6)$ & $1.1(0.9-1.4)$ & $1.0(0.8-1.3)$ \\
\hline
\end{tabular}

\begin{tabular}{|c|c|c|c|}
\hline Information source & $\begin{array}{l}\text { Did not use or } \\
\text { received no information } \\
\text { from it, } n(\%)\end{array}$ & $\begin{array}{l}\text { Used it and } \\
\text { received a little } \\
\text { information, } n(\%)\end{array}$ & $\begin{array}{c}\text { Used it and received } \\
\text { a lot of information } \\
n(\%)\end{array}$ \\
\hline \multicolumn{4}{|l|}{ Use of any British media source } \\
\hline Television & $101(34.2)$ & $119(40.3)$ & $75(25.4)$ \\
\hline Radio & $224(75.9)$ & $49(16.6)$ & $22(7.5)$ \\
\hline Newspapers & $151(51.2)$ & $97(32.9)$ & $47(15.9)$ \\
\hline Mainstream news websites & $32(10.8)$ & $128(43.4)$ & $135(45.8)$ \\
\hline Computed variable & $11(3.7)$ & $146(49.5)$ & $138(46.8)$ \\
\hline \multicolumn{4}{|l|}{ Use of any Japanese media source } \\
\hline Television & $51(17.3)$ & $131(44.4)$ & $113(38.3)$ \\
\hline Radio & $258(87.5)$ & $28(9.5)$ & $9(3.1)$ \\
\hline Newspapers & $165(55.0)$ & $96(32.5)$ & $34(11.5)$ \\
\hline Mainstream news websites & $91(30.8)$ & $123(41.7)$ & $81(27.5)$ \\
\hline Computed variable & $21(7.1)$ & $143(48.5)$ & $131(44.4)$ \\
\hline \multicolumn{4}{|l|}{ Use of any British government internet source } \\
\hline Contacting someone from the British Embassy or Foreign Office & $191(64.7)$ & $81(27.5)$ & $23(7.8)$ \\
\hline Teleconference with British chief scientific advisor & $198(67.1)$ & 45 (15.3) & $52(17.6)$ \\
\hline Reading British Embassy or Foreign Office Twitter or Facebook account & $169(57.3)$ & $76(25.8)$ & $50(17.0)$ \\
\hline Reading British Embassy or Foreign Office website or blog & $97(32.9)$ & $118(40)$ & $80(27.1)$ \\
\hline Reading another British government website or blog & $213(72.2)$ & $61(20.7)$ & $21(7.1)$ \\
\hline Computed variable & $75(25.4)$ & $154(52.2)$ & $66(22.4)$ \\
\hline \multicolumn{4}{|l|}{ Use of any informal interpersonal communication } \\
\hline Reading any official Japanese government website or blog & $194(65.8)$ & $76(25.8)$ & $25(8.5)$ \\
\hline Speaking with friends relatives or colleagues & $40(13.6)$ & 137 (46.4) & $118(40.0)$ \\
\hline Reading other Twitter or Facebook posts (not official government sources) & $176(59.7)$ & $70(23.7)$ & $49(16.6)$ \\
\hline Computed variable & $36(12.2)$ & $187(63.4)$ & $72(24.4)$ \\
\hline Speaking to your general practitioner & $284(96.3)$ & $11(3.7)$ & $0(0)$ \\
\hline
\end{tabular}




\begin{tabular}{|c|c|c|c|}
\hline Information source ${ }^{b}$ & $\begin{array}{c}\text { Distress } \\
\text { OR }(95 \% \mathrm{Cl})^{\mathrm{C}}\end{array}$ & $\begin{array}{c}\text { Anxiety } \\
\text { OR }(95 \% \mathrm{Cl})^{\mathrm{c}}\end{array}$ & $\begin{array}{c}\text { Anger } \\
\text { OR }(95 \% \mathrm{Cl})^{\mathrm{c}}\end{array}$ \\
\hline \multicolumn{4}{|l|}{ British media source } \\
\hline Any British media source & $0.6(0.3-1.2)$ & $1.5(0.9-2.6)$ & See interaction \\
\hline High credibility & No interaction & No interaction & $0.7(0.3-1.4)$ \\
\hline Low credibility & No interaction & No interaction & $2.8(1.2-6.8)$ \\
\hline Any Japanese media source & $0.6(0.3-1.2)$ & $1.0(0.6-1.7)$ & $1.5(0.9-2.6)$ \\
\hline Contacting someone from the British Embassy or Foreign Office ${ }^{d}$ & $1.1(0.5-2.0)$ & $1.0(0.6-1.7)$ & $1.3(0.8-2.3)$ \\
\hline Teleconference with the British chief scientific advisor $^{d}$ & See interaction & See interaction & $1.1(0.6-1.9)$ \\
\hline High credibility & $0.4(0.1-1.3)$ & $0.5(0.2-1.2)$ & No interaction \\
\hline Low credibility & $4.2(1.3-13.1)$ & $2.1(0.8-5.3)$ & No interaction \\
\hline Use of any British government internet media & $2.5(1.2-5.4)$ & $1.2(0.6-2.3)$ & $1.9(1.02-3.7)$ \\
\hline Reading any official Japanese government website or blog & $1.3(0.4-4.4)$ & $3.9(1.2-12.3)$ & $4.0(1.3-11.6)$ \\
\hline Use of any informal interpersonal communicatione & $0.9(0.4-2.0)$ & $1.2(0.6-2.2)$ & $2.0(1.1-3.6)$ \\
\hline \multicolumn{4}{|c|}{$\begin{array}{l}\text { a. Unless otherwise noted, the comparisons show the difference between people who received a lot of information from the source and those who reported receiving a little } \\
\text { information from it (the reference group). } \\
\text { b. Where a significant interaction with credibility existed, the results for people scoring high and low on credibility for that source are both shown. } \\
\text { c. Odds ratios are adjusted for gender, age, ethnicity, parental status, education and long-standing illness. } \\
\text { d. Comparing those who used source at all against those who did not (reference group). } \\
\text { e. Credibility was not measured for informal interpersonal communication. }\end{array}$} \\
\hline
\end{tabular}

perform to reduce any risk. Information was seen as useful for those in Japan and as reassurance for friends and relatives in Britain. Language barriers were cited as one factor encouraging a reliance on information from the Embassy, but many people were also keen to obtain information from the Embassy because of an expectation that it would be trustworthy and factual. For those who praised the FCO, comments such as '[they] were a trusted source of information', 'the Embassy's strength was that it was neutral' and 'scientific facts, not gossip and rumour' were common. This was frequently contrasted with the 'fog of information and misinformation', 'panicky advice' and 'hiding the truth' that predominated elsewhere. In contrast, those who listed information provision as something the Embassy should improve either cited a belief that official British information was based on Japanese government statements which were 'probably playing down the dangers' or else suspected that the British government was not providing 'the full story in case it led to panic'. The importance of credibility also permeated comments about the teleconferences with the chief scientific advisor, which were noted by many as a particularly salient aspect of the information they received. Comments were largely complimentary, focusing on the importance of receiving an 'honest, unbiased, scientific assessment' from someone who 'knew his field well'. For others, however, the mismatch between their perceptions of the risk and the information in the teleconference led them to believe that the advice was 'downplaying the severity of Fukushima'. This caused concern for some (e.g. 'Far too upbeat ... This concerns me and is one reason I'm now leaving Japan with my wife').

Although the credibility of information from the Embassy drew participants to it, several pragmatic aspects of the information were also mentioned as beneficial or in need of improvement. The use of multiple channels to provide communication (including Facebook and Twitter) was praised by many participants, although the lack of regular email updates was criticised. This was particularly true for the substantial number of participants who felt that information was not updated regularly enough (e.g. 'Even a quick "there are no changes since yesterday" would have at least made us feel we weren't alone'). Speed in providing information was noted as important, together with ensuring it was communicated clearly. Consistency was also valued, with apparent contradictions or changes in the risk assessment worrying several participants. This related not only to advice from the British government, but also the perceived inconsistency in the advice produced by different embassies. Finally, some participants requested that independent information from the Embassy continue to be provided in the long term (e.g. 'We are still here! [We] thirst for objective outside advice').

In addition to information about the radiation, a second main theme in participant responses was their expectation of advice on whether to leave the country. Clarity was expected, but those commenting on it often reported being left uncertain (e.g. 'We were informed that we should consider leaving the country. I was left wondering if this constituted an evacuation order or not'). How people interpreted the advice served as a signal to them about the implied level of risk (e.g. 'They should not have precipitated a panic by advising British nationals to leave', 'They didn't panic and give extreme advice to leave').

Implied messages about the risk were also perceived in the decision to distribute iodine tablets (a third main theme). Although many respondents were reassured by their provision and cited these as their 'one thing that the British Embassy did well', others believed that the distribution signalled a worrying change in the official risk assessment and were 'contradictory to the Embassy's travel advisory regarding Tokyo being reasonably safe'. Better notification about tablets being available and improving distribution were also suggested as important.

The provision of reassurance emerged as a fourth theme in how the FCO and Embassy reacted. Effective reassurance was noted as stemming from three main sources. First, the Embassy appeared calm in its communications and actions (e.g. 'I saw that Embassy staff remained in Tokyo so surmised it can't be so bad'). Second, individual members of staff were praised for their care and assistance (e.g. 'That personal touch is so important in a disaster'). Finally, the knowledge that the Embassy was available and had the best interests of British nationals at heart was a third source (e.g. 'I felt safe that if I needed it I could get help').

Finally, although participants reported a range of reactions to the FCO's response to the disaster, those who mentioned the British media were unanimous in their condemnation of its 'scaremongering', 'appalling misconceptions' and 'crap journalism'. The final main theme to emerge consisted of requests that the FCO intervene to 'counteract the most extreme tabloid speculation'. 


\begin{tabular}{|c|c|}
\hline Theme & Main subthemes \\
\hline $\begin{array}{l}\text { Information about } \\
\text { radiation }\end{array}$ & $\begin{array}{l}\text { Lack of bias and authoritative collation } \\
\text { of existing information } \\
\text { Information for use in reassuring friends } \\
\text { and relatives at home } \\
\text { Provision of information in native language } \\
\text { Provision of independent scientific advice } \\
\text { Use of multiple channels } \\
\text { Provision of regular updates } \\
\text { Consistent, clear and quick messages } \\
\text { Consistency with other embassies } \\
\text { Information flow maintained over the long-term }\end{array}$ \\
\hline Provision of travel advice & $\begin{array}{l}\text { Clear advice on whether to evacuate } \\
\text { Implied messages about risk }\end{array}$ \\
\hline Provision of iodine tablets & $\begin{array}{l}\text { Equitable distribution } \\
\text { Implied messages about risk }\end{array}$ \\
\hline Appropriate reassurance & $\begin{array}{l}\text { Appearing calm } \\
\text { Helpful, empathetic staff members } \\
\text { Demonstrating care for British nationals }\end{array}$ \\
\hline $\begin{array}{l}\text { Contacting British } \\
\text { nationals }\end{array}$ & $\begin{array}{l}\text { Accurate record-keeping } \\
\text { Using multiple channels to make contact } \\
\text { Rapid response to contact from British nationals }\end{array}$ \\
\hline Assistance with travel & $\begin{array}{l}\text { Assistance with cost } \\
\text { Appropriateness of 'evacuation' arrangements } \\
\text { Help and information on travel within Japan }\end{array}$ \\
\hline $\begin{array}{l}\text { Intervene in media } \\
\text { over-reactions }\end{array}$ & No subthemes \\
\hline
\end{tabular}

\section{Discussion}

Disasters impact on all of those who come in contact with them. This is particularly true when radiation is involved, since its invisible nature and the lengthy delay between exposure and the onset of health effects can cause substantial uncertainty and worry. ${ }^{10,11}$ Although the psychological ramifications of a nuclear disaster can be severe among the citizens of the country it occurs in, the effect on foreign nationals is less well established. In our study, the psychological effects of the Fukushima nuclear accident were high among British nationals who had been in Japan at the time. Although most did not experience any negative psychological effect, about a third reported high levels of anger and anxiety when thinking about the incident, and 16\% experienced distress that was strongly predicted by disaster-related variables.

We identified several psychological predictors of these emotional reactions. Some were consistent with conventional accounts of how people perceive risk, such as associations with the perceived severity of exposure or the perceived risk to children and pregnant women. ${ }^{12}$ However, most predictors related to uncertainty, with feeling uncertain when thinking about the incident being the strongest predictor for all emotional outcomes. Cognitive variables that are linked to uncertainty also predicted emotional responses, such as perceiving the risk to be mysterious, retaining an element of doubt about how much exposure one had received, thinking that any health effects might remain hidden for years to come and having a stronger conviction that it is not possible to detect exposure. The importance of uncertainty was also supported by our qualitative results, with requests for more information, consistency, clarity and regular updates all suggesting a desire to understand the risk better.

Although reducing uncertainty might be achieved through good communication, this can be difficult to achieve. Our results indicate that obtaining information from government websites and listening to the chief scientific advisor's teleconferences were associated with greater levels of distress and anxiety. Causation is difficult to prove for these associations. Yet because the association with the chief scientific advisor's teleconference was only seen among people who perceived the British government to have low credibility, it seems unlikely that these effects are simply due to distressed people seeking out more information. Instead, our qualitative results suggest that some people may have become worried by what they perceived to be false reassurance from these sources. Anger, meanwhile, was associated with using the British media among those who felt it lacked credibility, reading British or Japanese government websites and using interpersonal communication. Discrepancies between the reported risk and the risk as perceived by the participant may also account for these associations. Ensuring that information does not increase distress and anxiety in future disasters may require communicators to explain any discrepancies between expert and lay perceptions of the risk.

Our results also suggest that individual differences in the way people process information about a disaster should be taken into account by those who organise an emergency response. Not everybody wants or feels able to consider in-depth information about a risk before forming a judgement about it. ${ }^{13}$ In our study, although people who engaged in systematic information processing tended to react with more anger when they perceived the effects of exposure to be severe, the associations between emotional response and believing that the exposure could not be detected or would result in hidden health effects were stronger among those who scored low for systematic processing. In future incidents, public health communicators could assist people who feel unable or unwilling to systematically engage with information by only providing detailed information to those who actively seek it, by advising other members of the public to avoid continual monitoring of media coverage about the disaster and by encouraging more people to engage in systematic information processing by helping them find and interpret information.

\section{Limitations}

Five caveats should be borne in mind for our study. First, type one and type two errors may be present in the results, with the small sample size preventing potentially important associations from being observed, while spurious associations may have been produced by the numerous statistical tests that we used.

Second, the cross-sectional nature of our data makes it difficult for us to infer causality. A prospective cohort study beginning soon after a major incident would provide a better understanding of causal pathways.

Third, our sample may have been biased, with people who felt they had something 'interesting' to say in our survey or who were particularly motivated by our charitable donation being more inclined to participate.

Fourth, our questionnaire did not ask participants why they were in Japan at the time of the disaster. As a result, we are unable to differentiate those who were permanent or semi-permanent residents of Japan from those who were there for a short visit. Important differences in risk perception may exist between these groups.

Finally, the application of the PATHS questionnaire to the Fukushima incident was not without problems, particularly in terms of specifying the duration and intensity of exposure that respondents should consider for the questions. Future users of the questionnaire should pay close attention to how they specify these items. 


\section{Implications}

In a future disaster involving a chemical, biological or radiological threat, reducing uncertainty about the risk remains the best way of reducing the emotional burden among the general public. However, the use of information to reduce uncertainty should be carefully planned. Some information will inevitably be produced by the government of the affected country, but foreign nationals are likely to turn to their own embassies for advice and information. Embassies must be ready to supplement their consular roles with the collation, authentication and dissemination of information, and to provide regular updates over an extended period of time. Care should be taken not to provide explicit reassurance in these updates unless it is supported by credible evidence, not only because trust may be damaged if the situation worsens, but also because reassurance may increase worry if it is perceived as being at odds with other information about the situation. Attention may also need to be paid to people who do not feel equipped to deal with information about the disaster, but who perceive themselves as being at risk. Advising such people to limit their exposure to media reporting and helping them to identify and understand good-quality information when they wish to do so may reduce levels of distress.

G. James Rubin, PhD, King's College London, Institute of Psychiatry, Department of Psychological Medicine, Weston Education Centre, London; Richard Amlôt, PhD, Health Protection Agency, Emergency Response Department, Salisbury, Wiltshire: simon wessely, MD, FRCPsych, Neil Greenberg, MD, FRCPsych, King's College London, Institute of Psychiatry, Department of Psychological Medicine, Weston Education Centre, London, UK

Correspondence: G. James Rubin, King's College London, Institute of Psychiatry, Department of Psychological Medicine, Weston Education Centre (P062), Cutcombe Road, London SE5 9RJ, UK. Email: gideon.rubin@kcl.ac.uk

First received 6 Mar 2012, final revision 31 May 2012, accepted 27 Jun 2012

\section{Funding}

This study was funded through a Career Development Fellowship awarded to G.J.R. by the National Institute for Health Research. The funders did not participate in the study design, data collection, analysis or interpretation, or in the preparation or submission of this paper.

\section{Acknowledgements}

We are grateful to our respondents for assisting with this research and to Ben Collins, John Simpson, Nick Gent (Health Protection Agency), Georgina Hurlock and Peter Connolly (FCO) for their assistance.

\section{References}

1 Smerecnik CMR, Mesters I, Candel MJJM, De Vries H, De Vries NK. Risk perception and information processing: the development and validation of a questionnaire to assess self-reported information processing. Risk Anal 2012; 32: $54-66$.

2 McComas KA, Trumbo $\mathrm{CW}$. Source credibility in environmental health-risk controversies: application of Meyer's credibility index. Risk Anal 2002; 21: 467-80.

3 Goldberg D. General Health Questionnaire (GHQ-12). nferNelson, 1992.

4 Spielberger CD. STAXI-2: State-Trait Anger Expression Inventory-2. Psychological Assessment Resources, 1999.

5 Marteau TM, Becker $\mathrm{H}$. The development of a six-item short-form of the state scale of the Speilberger state-trait anxiety inventory (STAI). Br J Clin Psychol 1992; 31: 301-6.

6 Rubin GJ, Amlôt R, Page L, Wessely S. Public perceptions, anxiety and behavioural change in relation to the swine flu outbreak: a cross-sectional telephone survey. BMJ 2009; 339: b2651.

7 Roussos A, Goenjian AK, Steinberg AM, Sotiropoulou C, Kakaki M, Kabakos C, et al. Posttraumatic stress and depressive reactions among children and adolesents after the 1999 earthquake in Ano Liosia, Greece. Am J Psychiatry 2005; 162: 530-7.

8 Rubin GJ, Amlôt R, Page L, Pearce J, Wessely S. Assessing Perceptions AbouT Hazardous Substances: The PATHS questionnaire. Journal of Health Psychology 2012; in press.

9 Meyer P. Defining and measuring credibility of newspapers: developing an index. Journal Q 1988; 65: 567-74.

10 Bromet EJ. Lessons learned from radiation disasters. World Psychiatry 2011; 10: 83-4.

11 Vyner HM. The psychological dimensions of health care for patients exposed to radiation and the other invisible environmental contaminants. Soc Sci Med 1988; 27: 1097-103.

12 Slovic P. Perception of risk. Science 1987; 236: 280-5.

13 Trumbo CW. Heuristic-systematic information processing and risk judgement. Risk Anal 1999; 19: 391-400. 